\title{
Fire-spotting generated fires. Part I: The role of atmospheric stability
}

\author{
Vera N. Egorova ${ }^{\mathrm{a}, *}$, Andrea Trucchia ${ }^{\mathrm{a}}$, Gianni Pagnini ${ }^{\mathrm{a}, \mathrm{b}}$ \\ ${ }^{a}$ BCAM - Basque Center for Applied Mathematics, Alameda de Mazarredo 14, E-48009 \\ Bilbao, Basque Country - Spain \\ ${ }^{b}$ Ikerbasque - Basque Foundation for Science, Calle de María Díaz de Haro 3, E-48013 \\ Bilbao, Basque Country - Spain
}

\begin{abstract}
This is the first part of two papers concerning fire-spotting generated fires. In this part we deal with the impact of macroscale factors, such as the atmospheric stability, and in the second part we deal with mesoscale factors, such as the flame geometry. For this study we adopt an approach where the motion of the front is split into a drifting part and a fluctuating part. The drifting part, that can be provided by choosing an existing operational model, is here based on the levelset method in analogy with WRF-SFIRE model. The fluctuating part, that is the result of a comprehensive statistical description of the physics of the system and includes the random effects, is here physically parametrized to include turbulent hot-air transport and firebrand landing distance. In order to highlight the net effects of the random contributions due to turbulence and firebrand flying, a simplified model without fire-atmosphere coupling is considered. Numerical simulations show that the atmospheric stability is an important factor for wildfire propagation. In particular, unstable conditions boost the number of fire-spotting generated fires at small elapsed times as well as the strength of turbulence leading to rapid merging and the formation of unburned islands surrounded by the fire. Stability conditions have then an effect on the risk and the management associated to fire-spotting generated fires. In fact, with stable conditions (corresponding for example to the night-time) the turbulence is not strong enough to merge the fires and, at large elapsed times, this results into a higher number of independent fires but lower burned area with respect to un-
\end{abstract}

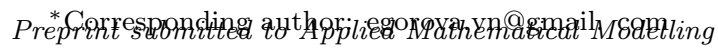


stable conditions (corresponding for example to the day-time) when the push of turbulence leads to faster merging resulting into a lower number of independent fires but higher burned area. Finally, with stable conditions less fire fronts need to be managed at short time, but more fire fronts need to be managed than with unstable conditions that however show a higher risk because of the merging of independent fires.

Keywords: Wildfire, Fire-spotting, Atmospheric stability

\section{Introduction}

Wildfire is a worldwide phenomenon that takes place in any vegetated area regardless of national fire-fighting management strategies and causing significant damages for the environment, properties and human lives [1]. Wildfires have a strong social and economical impact calling for a deeper understanding of their behaviour for controlling the risk and managing their suppression [2].

Wildfire propagation is a multiscale phenomenon involving processes from the scale of the combustion chemistry to the fire-atmosphere coupling including effects due, for example, to the topography and to the flame geometry [3, 4]. One

20 of the key aspects of fire propagation is the so-called fire-spotting [5]. It occurs when burning embers tear off from the main fuel source and cause new independent ignitions. Hence the hazard increases because the fire spread accelerates bringing harmful consequences. Fire-spotting has an unpredictable nature so it is a challenging issue in wildfire science. In the present pair of papers, we study the role for the emergence of fire-spotting phenomena and the ignition of secondary fires of both a macroscale factor as the atmospheric stability, in the first part, and of a mescoscale factor as the flame length, in the second part.

Because of the interactions among scales, we follow a concurrent multiscale modelling, that means estimating parameters related to aspects occurring in a very large range of scales and implementing them into the model for the macroscopic fire perimeter, see Figure 1. The adopted modelling approach [6, 7] 
is based on the idea to split the motion of the front into a drifting part and a fluctuating part. The drifting part, that can be provided by choosing an existing operational model, is here based on the level-set method [8, 9] in analogy with WRF-SFIRE model [10. The fluctuating part, that is the result of a comprehensive statistical description of the physics of the system and includes the random effects, is here physically parametrized to include turbulent hot-air transport and firebrand landing distance.

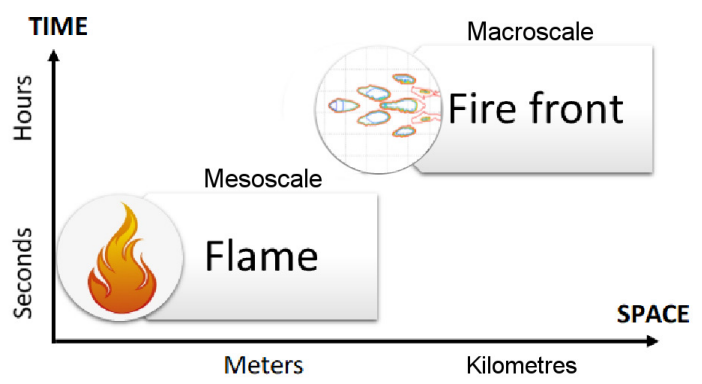

Figure 1: Multiscale modelling of wildfires.

Despite fire-spotting is responsible for the increase and unpredictability of 40 fire danger, its understanding remains limited. Among various stages and factors that influence on fire-spotting, firebrand lofting is a highly stochastic process that has a strong impact on the downwind transport of firebrands. Therefore, the firebrand transportation is of the special interest to the researchers and has been extensively studied. Most of the firebrand transport models are based on the mass and shape of particles, plume characteristics and ambient factors. In Tarifa et al. [11] the travel distance of the firebrand is studied in terms of firebrand size, density and shape. Albini's model [12, 13] is focused on the estimation of the maximum distance for the secondary fire basing on the linear relationship between the burning area and the mean wind. To date, many fire spread models, such as Farsite [14] or Prometheus proposed in [15], are extensions of the Albini's model. However, application of these models to the fire-spotting problem has not been promising as no any function for the ignition 
probability is considered. In this regard, there are recent works that analyse the generation and downwind distribution of firebrands (see [16, 17, 18]). Other stochastic models of fire-spotting are also proposed in [19, 20, 21.

Recent trend in operational codes is model based on coupling the wildfire propagation with the atmospheric models, as it is implemented in WRF-Sfire [10]. Such models provide a better representation of initial and concurrent atmospheric conditions, but do not take into account random effects of the firespotting. Lack of comprehensive and versatile approach for implementation of fire-spotting routines into operational models motivates the research for a physical parametrisation of the fire-spotting. In [22] an independent post-processing routine that can be implemented to any existing wildfire propagation model has been proposed. In the present work we proceed with the study presented in Ref. 22 by investigating in the first part the role of the atmospheric stability and in the second part the role of the flame length. These two aspects of the proposed model have different nature and different scales, thus in order to localise and estimate the impact of each of the parameter the paper is splitted into two parts.

At the macroscopic scale, fire-spotting is affected by atmospheric conditions. In particular, we plug the depth of the atmospheric boundary layer (ABL), that is related to the atmospheric stability, into the estimation of the smoke-injection height including the uplift against the atmospheric stratification and the plume widening due to entrainment of the surrounding air 23 . Later, we estimate the firebrand-injection height as an approximated lift for inertial particles flowing into the fire plume.

At the mesoscopic scale, fire-spotting is affected strongly by the fire intensity and by the flame characteristics, fire intensity being related to many aspects of the flame geometry, see [24] and references therein.

80 The aim of the research is to highlight the role of a macroscopic factor and the one of that of a mesoscopic factor for characterizing fire-spotting in order to provide information on favourable configurations for the occurrence of firespotting and the associated risk. Implicit connection between the atmospheric 
stability and fire propagation allows the modelling of various scenarios taking into account times of day of wildfire. Moreover, an important improvement is proposed in establishing the range of unburned terrain where the secondary fires may occur, which allows to model different firebrand shower scenarios. The viability of this improvement is shown by numerical examples.

The rest of the paper is organized as follows. In Section 2 we propose a parametrisation of the jump-length distribution of firebrand extending previous results and in Section 3 we briefly present the fire propagation model. In particular, fire-spotting, as well as turbulent heat transport, are implemented into the model through a density function combining turbulent diffusion and firebrand jumps. The uniqueness of this density function is studied in Section 4 and some

95 remarks in view of the present application are reported. Numerical results are presented and discussed in Section 5 , and conclusions are given in Section 6 .

\section{Parametrisation of the firebrand landing distribution}

The aim of this paper is the investigation of the role of the atmospheric stability on the generation of independent secondary fires due to firebrands emissions. The downwind landing distribution of firebrands $q(\ell)$ is here assumed to be lognormal following [18, 25],

$$
q(\ell)=\frac{1}{\sqrt{2 \pi} \sigma \ell} \exp \frac{-(\ln \ell / \mu)^{2}}{2 \sigma^{2}},
$$

where $\mu$ is the ratio between the square of the mean of landing distance $\ell$ and its standard deviation $\sigma$.

In order to highlight the net effects of the random contributions due to turbulence and firebrand flying, a simple model without the coupling between the fire and the weather forecast is considered. Atmospheric conditions are used to parametrize the turbulent diffusion coefficient of hot air and the landing distribution of firebrands.

A description of the fire-spotting phenomenon in terms of the fire intensity, wind velocity and fuel characteristics is given in Ref. [22] and it has been 
applied to study wildfire propagation model in Ref. [6]. The parametrisation stated in Ref. 222 is based on the assumption that each firebrand is spherical with constant size. Following [22, the lognormal parameters $\mu$ and $\sigma$ in (1) result to be

$$
\mu=H\left(\frac{3 \rho C_{\mathrm{d}}}{2 \rho_{\mathrm{f}}}\right)^{1 / 2}, \quad \sigma=\frac{1}{z_{\mathrm{p}}} \ln \left(\frac{U}{\sqrt{r g}}\right),
$$

where $U$ is the wind speed and $H$ is the maximum loftable height of firebrands. The dimensionless ratio $\mathrm{Fr}=U^{2} /(\mathrm{rg})$ can be understood as an analogue of the Froude number, that is a measure of the balance between the inertial and the gravitational forces experienced by the burning ember. In terms of the Froude number the standard deviation $\sigma$ can be written as follows

$$
\sigma=\frac{1}{2 z_{\mathrm{p}}} \ln F r .
$$

The injection height of firebrand is a fraction of the injection height of the smoke $H_{\text {smoke }}$, i.e., $H=\bar{\nu} H_{\text {smoke }}<H_{\text {smoke. }}$. In the following we consider $\bar{\nu}=$ 0.4, see Appendix for this estimation.

The injection height can be described in terms of buoyancy frequency, or Brünt-Väsäla frequency $N^{2}[23$. It is used in meteorology as a measure of atmospheric stratification and stability. Positive $N^{2}$ characterizes a stable boundary layer (SBL). If the potential temperature is uniform with height, the displaced parcel experiences no buoyancy force and will remain at its new location. Such a layer of air is neutrally stable. If the potential temperature increases with height, a parcel displaced upwards (downwards) experiences a negative (positive) restoring force and will tend to return to its equilibrium level. Usually it can be observed during the night, therefore, it is also known as nocturnal boundary layer. In contrast, atmosphere is considered to be unstable, if the potential temperature decreases with height, and a displaced parcel would experience a force in the direction of the displacement. Unstable atmosphere is described by negative values of $N^{2}$ that may cause the appearance of complex numbers in the formulation of the injection height. In order to avoid such problems, general formulation has been proposed in 23 . by using the free troposphere value $N_{\mathrm{FT}}^{2}$ 
[23] and letting a part of the ABL $(\alpha<1)$ be considered as "freely" passed. Here we use the following generic formula for the injection height of the smoke

$$
H_{\mathrm{smoke}}=\alpha H_{\mathrm{ABL}}+\beta\left(\frac{I_{\mathrm{f}}}{d P_{f_{0}}}\right)^{\zeta} \exp \left(-\frac{\delta_{\mathrm{FT}} N_{\mathrm{FT}}^{2}}{N_{0}^{2}}\right),
$$

where $I_{\mathrm{f}}$ is the fire intensity, $H_{\mathrm{ABL}}$ is the height of ABL. Parameters are constrained by: $\alpha<1, \beta>0, \zeta<0.5$ and $\delta_{\mathrm{FT}} \geq 0$ [23. Hence, the stability conditions of the atmosphere enter into the parametrisation through the height of the ABL. Note that $H_{\mathrm{ABL}}$ has an impact not only in the fire-spotting parametrisation, but it is also an important parameter for turbulent diffusion also, as it will be shown below.

On the basis of the maximum loftable height (4), the maximum travel distance can be defined as follows [26,

$$
\ell_{\max }=H\left(\frac{3 \rho C_{\mathrm{d}} F r}{2 \rho_{\mathrm{f}}}\right)^{1 / 2},
$$

that by substitution of (2) leads to

$$
\ell_{\max }=\mu \exp \left(z_{\mathrm{p}} \sigma\right)
$$

In fact, formulation (4), as well as (5), takes into account stable, neutral and unstable atmosphere. Thus, the model could be used to describe wildfire behaviour at any daytime.

The wind velocity in (2) is the projection of the vector of wind to the vector $\Phi$ from some point of the computational domain to the point where the PDF is computed. Therefore, denoting by $\omega$ the angle between the wind direction and the vector $\Phi$, the expression of $\sigma$ in 2 becomes

$$
\sigma_{\omega}=\frac{1}{2 z_{\mathrm{p}}} \ln \left[\frac{(U \cos \omega)^{2}}{r g}\right] .
$$

Hence, $\sigma_{\omega}$ depends not only on the wind velocity, but also on the position of the considered point. We assume downwind fire-spotting, so $-\pi / 2 \leq \omega \leq$ $140 \pi / 2$. If angle $\omega$ tends to $\pi / 2, \sigma$ becomes negative: that leads to small value of the maximum landing distance. In the limit case, firebrands fall down to 


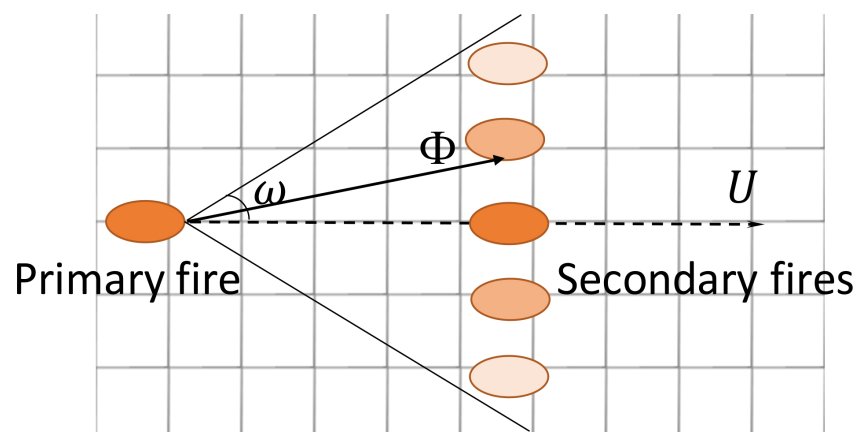

Figure 2: Sketch of the vector $\Phi$ and the angle $\omega$ and the generation of multiple fire-spotting.

already burned terrain and there is no fire-spotting effect. Thus, consideration of negative values of $\sigma_{\omega}$ is not expedient. From the other side, for $\omega$ close to zero, vector $\Phi$ has the same direction as the mean wind vector (see Figure 2 ) and the travel distance is at its peak. We establish limit angle $\omega_{0}$ in order to reduce computational cost, such that the fire-spotting distribution is calculated for $\cos \omega>\cos \omega_{0}$.

The minimum travel distance can be defined in terms of the parameters $\mu$ and $\sigma$ as follows

$$
\ell_{0}=\mu \exp \left(z_{\mathrm{p}}^{0} \sigma\right)=H\left(\frac{3 \rho C_{\mathrm{d}}}{2 \rho_{\mathrm{f}}}\right)^{1 / 2} \frac{U \cos \omega_{0}}{\sqrt{r g}}
$$

one gets

$$
\cos \omega_{0}=\left(\frac{U}{\sqrt{r g}}\right)^{\frac{z_{\mathrm{p}}^{0}}{z_{\mathrm{p}}}-1}=F r^{\frac{z_{\mathrm{p}}^{0}}{z_{\mathrm{p}}}-1},
$$

where $z_{\mathrm{p}}^{0}$ is the percentile of the lognormal distribution corresponding to the equality:

$$
q\left(\ell_{\max }\right)=q\left(\ell_{0}\right) .
$$

By plugging-in (5) and (8) into $(10)$ it follows

$$
\left(z_{\mathrm{p}}^{0}\right)^{2}+2 \sigma z_{\mathrm{p}}^{0}=z_{\mathrm{p}}^{2}+2 \sigma z_{\mathrm{p}}
$$

that has two solutions: $z_{\mathrm{p}}$, that gives the maximum travel distance and the 
following

$$
z_{\mathrm{p}}^{0}=-z_{\mathrm{p}}-2 \sigma
$$

$$
\cos \omega \geq \max \left\{\frac{1}{\kappa}, F r^{-1 / 2}\right\}
$$

Relation (14) between the critical angle $\omega_{0}$ and the range of the possible travel distances represented by $\kappa$ may also be interpreted as inverse proportionality of the arc and the radius in a sector of the fixed area. When $\omega$ tends to $\pi / 2$, the projection of the wind approximates to zero, that corresponds to the situation when the firebrand falls down into the burning area. Thus, the reasonable constrain to reduce computational efforts reads as follows

Atmospheric stability considered above influences not only on the firebrand transport, but also on the turbulence. ABL is turbulent itself, but in presence of fire, the turbulent heat transfer plays an important role in wildfire propagation and thus, has to be considered. We introduce the turbulent diffusion coefficient $D$, that is estimated by the following formula [7]

$$
D \simeq \chi\left(0.1 R a^{\beta_{\mathrm{D}}}-1\right), \text { where } R a=\gamma_{\mathrm{D}} \Delta T g H_{\mathrm{ABL}}^{3} \nu^{-1} \chi^{-1},
$$

where $\chi$ is the thermal diffusivity of the air at ambient temperature, $R a$ is the Rayleigh number, $\gamma_{\mathrm{D}}$ is the thermal expansion coefficient, $\Delta T$ is the tempera- 

kinematic viscosity. The vertical dimension of the convection cell can be estimated by the smoke-injection height given by (4), that is less than $H_{\mathrm{ABL}}$, while in the absence of fire the atmospheric flow is turbulent in the whole ABL [27. Taking into account both facts, we assume that the height of the convection difference between the flame and the air of the top of the ABL, that is much lower than the flame temperature. Thus, the value of $\Delta T$ is close to the flame temperature.

The average characteristics of a fire flame are $1 \mathrm{~m}$ in height and $1100 \mathrm{~K}$, or of the igniting sector of secondary fires, is implemented as a post-processing routine into a wildfire simulator based on the level-set method embodying the improvement and the novelty of the present model with respect to the previous formulation [6, 7, 22. A brief description of the modelling approach is presented 195 more, in temperature. Experimental data presented in Ref. 28] and references therein, the temperature of the flame varies from $800 \mathrm{~K}$ to $920 \mathrm{~K}$. Under extreme conditions, the flame height may be about $50 \mathrm{~m}$, or more, and the temperature exceeds $1500 \mathrm{~K}$ (see http://wildfiretoday.com). For example, for the pinus caribea (pine) litter the flame height was $1 \mathrm{~m}$ and the maximum temperature observed $1473 \mathrm{~K} 29$.

Parameter $\beta_{\mathrm{D}}$ is the exponent of the power law relating the Nusselt number $N u$ and the Rayleigh number $R a$, i.e., $N u=0.1 R a^{\beta_{\mathrm{D}}}$ 30, 31. In particular, in Ref. 30] the authors report that with $R a>5 \cdot 10^{7}$ it holds $N u=0.146 R a^{0.299}$, and in Ref. [31] the authors report that with $10^{8}<R a<10^{10}$ the $N u$ and $R a$ correlation display a nearly constant and of magnitude value $\beta_{\mathrm{D}}=0.31$. For higher $R a$ it saturates at around $1 / 3$ in the range of $R a$ for which the Boussinesq limit is valid strictly. Previous experimental results suggested $\beta_{\mathrm{D}} \approx 2 / 7$ instead of $1 / 3\left[32\right.$. In the following we use $\beta_{\mathrm{D}}=0.3$.

This physical parametrisation of the firebrand landing distribution, together with the lower boundary of the standard deviation and the selection criterion in the following Section. 


\section{Fire propagation model}

The motion of wildfire front can be split into a drifting part and a fluctuating part. After the splitting, the drifting part can be treated by any existing method, such as the LSM that is chosen for the present study in analogy with that is independent of the drifting part, describes the effects of turbulence and fire-spotting as random phenomena and they are implemented into the model through a post-processing routine [6, 7]. Here we provide a short description of this model.

For a given computational domain $S$ the fire front contour is represented by a closed curve $\Gamma$. The region bounded by $\Gamma$ is denoted by $\Omega$ and represents the burnt area. Let $X=\bar{X}+\eta$ be the stochastic trajectory of the active fire point, where $\bar{X}$ is the drifting part obtained from a wildfire propagation model (e.g., the LSM or the DEVs), and $\eta$ is the random noise characterised by its probability density function (PDF). In wildfire propagation, the fluctuating part can have a non-zero mean due to, for instance, travel distance of a firebrand, such that the drifting part does not correspond to the average motion [6]. Let us introduce an indicator function $\phi(\mathbf{x}, t)$ :

$$
\phi(\mathbf{x}, t)= \begin{cases}1, & \mathbf{x} \in \Omega(t), \\ 0, & \text { othervise }\end{cases}
$$

205

Let $\gamma: S \times[0, \infty) \rightarrow \mathbb{R}$ be a level-set function, such that for some fixed $\gamma^{*}$ at the moment $t$ the fire front can be described by $\Gamma(t)=\left\{\mathbf{x} \in S \mid \gamma(\mathbf{x}, t)=\gamma^{*}\right\}$. If $\gamma(\mathbf{x}, t)>\gamma^{*}$, then the ignition is observed at the point $\mathbf{x}$. The level-set function $\gamma(\mathbf{x}, t)$ evolves according to the following ordinary level-set equation

$$
\frac{\partial \gamma}{\partial t}=V_{\mathrm{ROS}}(\mathbf{x}, t)\|\nabla \gamma\|,
$$

where $V_{\mathrm{ROS}}(\mathbf{x}, t)$ is the rate of spread (ROS) of the fire front. The ROS value 210 depends on many factors, such as the intensity and direction of the wind, fuel conditions, etc. 
The wildfire propagation has a significant random component caused by the turbulent heat transfer and fire-spotting phenomena. The random front contour can be defined by the effective indicator function $[\underline{6}$ :

$$
\phi_{\mathrm{e}}(\mathbf{x}, t)=\int_{S} \phi(\overline{\mathbf{x}}, t) f(\mathbf{x} ; t \mid \overline{\mathbf{x}}) d \overline{\mathbf{x}}=\int_{\Omega(t)} f(\mathbf{x} ; t \mid \overline{\mathbf{x}}) d \overline{\mathbf{x}},
$$

where $f(\mathbf{x} ; t \mid \overline{\mathbf{x}})$ is the PDF that accounts for turbulence and fire-spotting effects. Note that the point is labelled as burnt, if $\phi_{\mathrm{e}}(\mathbf{x}, t)$ exceeds some threshold value $\phi_{\mathrm{e}}^{t h}$.

In accordance with the Reynolds transport theorem, the evolution of the effective indicator function $\phi_{\mathrm{e}}(\mathbf{x}, t)$ takes the form

$$
\frac{\partial \phi_{\mathrm{e}}}{\partial t}=\int_{\Omega(t)} \frac{\partial f}{\partial t} d \overline{\mathbf{x}}+\int_{\Omega(t)} \nabla_{\overline{\mathbf{x}}}[V(\overline{\mathbf{x}}, t) f(\mathbf{x} ; t \mid \overline{\mathbf{x}})] d \overline{\mathbf{x}} .
$$

When the evolution equation for the PDF $f(\mathbf{x} ; t \mid \overline{\mathbf{x}})$ takes the form

$$
\frac{\partial f}{\partial t}=\epsilon f
$$

where $\epsilon=\epsilon(\mathbf{x})$ is a generic evolution operator, equation 20 can be rewritten in the following form

$$
\frac{\partial \phi_{\mathrm{e}}}{\partial t}=\epsilon \phi_{\mathrm{e}}+\int_{\Omega(t)} \nabla_{\overline{\mathbf{x}}}[V(\overline{\mathbf{x}}, t) f(\mathbf{x} ; t \mid \overline{\mathbf{x}})] d \overline{\mathbf{x}}
$$

In 22 the front-line velocity is controlled by the ROS, while random process, such as turbulence and fire-spotting, are modelled by modifying PDF. Thus, if $f(\mathbf{x} ; t \mid \overline{\mathbf{x}})=\delta(\mathbf{x}-\overline{\mathbf{x}})$, equation 22 reduces to the deterministic case described 225 by 18 .

Assuming that the downwind phenomenon of fire-spotting is given by the turbulence and fire-spotting, the random process handled by $f(\mathbf{x} ; t \mid \overline{\mathbf{x}})$ in 22 can be defined as follows

$$
f(\mathbf{x} ; t \mid \overline{\mathbf{x}})= \begin{cases}\int_{0}^{\infty} G(\mathbf{x}-\overline{\mathbf{x}}-\ell \mathbf{n} ; t) q(\ell) d \ell, & \text { if } \cos \omega>\cos \omega_{0} \\ G(\mathbf{x}-\overline{\mathbf{x}} ; t), & \text { otherwise }\end{cases}
$$


where $q(\ell)$ is a lognormal distribution (1) with parameters $\mu$ and $\sigma_{\omega}$, given in (2) and (7), and $\cos \omega_{0}$ is defined by (15).

As it is mentioned above, the point is marked as burned if the effective indicator exceeds the threshold value $\phi_{\mathrm{e}}^{t h}$. However, there exists an additional criterion associated with an ignition delay due to pre-heating action of the hot air or to the landing of firebrands. This delay is considered as a heating-beforeburning mechanism due to the hot air in Refs. [34, 35, and it can be generalized to include fire-spotting in [6]. Since the fuel can burn because of two pathways - hot-air heating and firebrand landing, the resistance analogy suggests that the resulting ignition delay can be approximatively computed as resistances acting in parallel. Let $\tau_{\mathrm{h}}$ and $\tau_{\mathrm{f}}$ be the ignition delay due to hot air and firebrands landing, respectively, the joint ignition delay $\tau$ is

$$
\frac{1}{\tau}=\frac{1}{\tau_{\mathrm{h}}}+\frac{1}{\tau_{\mathrm{f}}}=\frac{\tau_{\mathrm{h}}+\tau_{\mathrm{f}}}{\tau_{\mathrm{h}} \tau_{\mathrm{f}}} .
$$

The heating-before-burning mechanism is depicted as the persistence in time of the effective fire front, i.e.,

$$
\psi(\mathbf{x}, t)=\int_{0}^{t} \phi_{\mathrm{e}}(\mathbf{x}, \eta) \frac{d \eta}{\tau}
$$

where $\psi(\mathbf{x}, 0)=0$ corresponds to the unburned initial condition, and ignition at the point $\mathbf{x}$ at the moment $t$ occurs, if $\psi(\mathbf{x}, t)=1$.

\section{Study of uniqueness}

This section deals with the uniqueness of the PDF $f(\mathbf{x} ; t \mid \overline{\mathbf{x}})$ in 23 for the

effective indicator function $\phi_{\mathrm{e}}(\mathbf{x}, t)$ in 19$)$, on the basis of the Radon-Nikodym theorem ([36], p. 233). We show that the choice of the PDF $f$ is not unique for a given $\phi_{\mathrm{e}}$ and this is positive for modelling.

In fact, consider two PDFs $f_{1}$ and $f_{2}$ with $\mathbf{x}, \overline{\mathbf{x}} \in R^{N}$ such that, in analogy with the Gaussian, $f_{1}=f_{1}(|\mathbf{x}-\overline{\mathbf{x}}| ; t)$ and $f_{2}=f_{2}(|\mathbf{x}-\overline{\mathbf{x}}| ; t)$, then

$$
\int f_{1} d \mathbf{x}=\int f_{1} d \overline{\mathbf{x}}, \quad \int f_{2} d \mathbf{x}=\int f_{2} d \overline{\mathbf{x}} .
$$


In general, there exists always a pair of $\operatorname{PDFs} f_{1}$ and $f_{2}$ that may be identical in the bulk but differ in their tails and it holds

$$
\begin{cases}f_{1}=f_{2}, & \overline{\mathbf{x}} \in \Omega, \\ f_{1} \neq f_{2}, & \overline{\mathbf{x}} \notin \Omega .\end{cases}
$$

Hence, with reference to the observable $\phi_{\mathrm{e}}$ defined in $(19)$, the same output can be obtained by different PDFs.

Thus, in terms of the model, the following statement can be formulated: the same burned area described by the effective indicator function $\phi_{\mathrm{e}}(\mathbf{x}, t)$ can be generated by different PDFs $f(\mathbf{x} ; t \mid \overline{\mathbf{x}})$. This is an important issue, and two remarks are in order in view of the practical purposes of the modelling approach.

The first remark concerns the positive fact that this allows for modelling a complex topology of the burning area by a simple PDF through a suitable choice of the parameters. This is the case, for example, when the vegetation inside the burned area is completely burned out and the combustion ceases. Thus, the burning area takes the form of crown or ring, since the ignitions are observed only at the boundary of the area. However, fire propagation can be still modelled by a PDF for the entire area with properly calibrated parameters.

The second remark concerns the fact that the domain $\Omega$ in $\sqrt{19}$ grows in time, and this makes artificial the determination of a pair of PDFs that meets (27) for a time-dependent domain. Since we are interested in the applications, this means that the non-uniqueness holds de facto for short times and the uniqueness follows de facto for large times.

\section{Results and discussion}

In this section numerical simulations with different atmospheric stability conditions, i.e., varying the height of the ABL, are described and analysed. The statistical data of wildfires in Spain from 2012 to 2017 are also reported in order to convey the idea that wildfires grow differently under different boundary layer conditions. 
In all the simulations we consider a constant wind $U=4.47 \mathrm{~ms}^{-1}$, that corresponds to the average wind speed in Madrid during 2017 (https:/ www.worldweatheronline.com/madridweather-averages/madrid/es.aspx), and fire intensity $I_{\mathrm{f}}=20 \mathrm{MWm}^{-1}$. The changes in the values of $H_{\mathrm{ABL}}$ have a strong effect on the values of the maximum travel distance of firebrands $\ell_{\max }$ as well as on the values of the turbulent diffusion coefficient $D$, see Table 1 and Figure 3 . The PDF of firebrand landing distance for different values of $H_{\mathrm{ABL}}$ is plotted in Figure 6 .

From formula (5) we have that $\ell_{\max }$ depends linearly on $H_{\mathrm{ABL}}$ and, due to the lognormal distribution of landing distance, the resulting changes in $\ell_{\max }$ do not strongly affect fire-spotting. From $(16)$ we have that the turbulent diffusion coefficient depends on $H_{\mathrm{ABL}}$ with the third power and the resulting changes affects strongly the propagation of the fire with an important effect on the merging of the secondary fires generated by the fire-spotting. In fact, during stable conditions a larger number of fires with respect to unstable conditions is observed but at the same time a lower burned area is computed, showing that in unstable conditions the turbulent heat transport is stronger with the double effects of a faster fire propagation and a more efficient merging.

\begin{tabular}{|c|rrr|rrrr|}
\hline & \multicolumn{3}{|c|}{ Case 1: Stable } & \multicolumn{4}{c|}{ Case 2: Unstable } \\
$H_{\mathrm{ABL}}, \mathrm{m}$ & 100 & 200 & 400 & 800 & 1000 & 1200 & 2000 \\
$\mu, \mathrm{m}$ & 4.0325 & 4.3879 & 5.0985 & 6.5198 & 7.2304 & 7.9411 & 10.7836 \\
$D, \mathrm{~m}^{2} \mathrm{~s}^{-1}$ & 0.0533 & 0.0995 & 0.1858 & 0.3467 & 0.4238 & 0.4993 & 0.7908 \\
\hline
\end{tabular}

Table 1: Effects of atmospheric stability on the fire-spotting parameters.

Case 1. Stable atmosphere: $H_{\mathrm{ABL}}$ varies from $100 \mathrm{~m}$ to $500 \mathrm{~m}$.

If an air parcel displaced from the original height returns to the original height, then the the atmosphere is said to be in stable condition. the stable boundary layer forms at night over land, also known as nocturnal boundary layer, which grows to depths of about 100 to $500 \mathrm{~m}$ [27. Due to the stability condition, clouds lire in layers and winds are steady and light. The smoke 

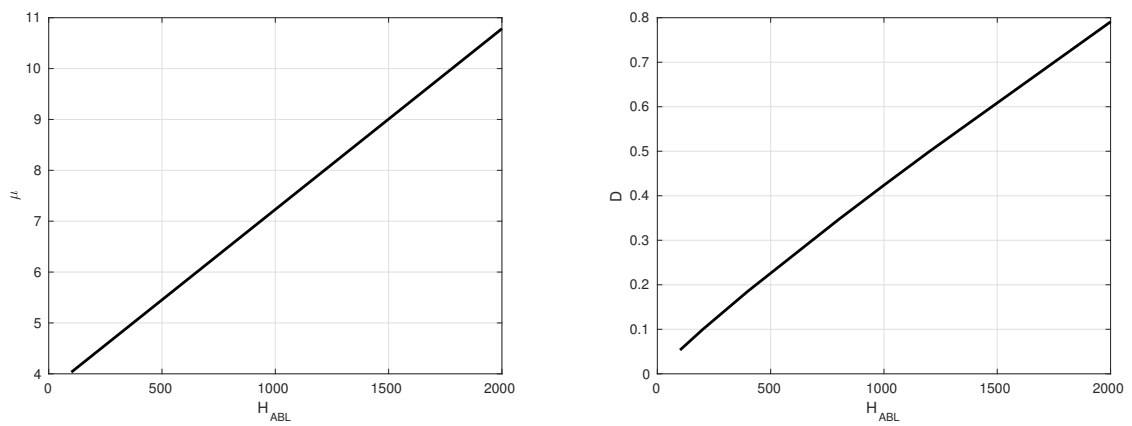

Figure 3: Dependencies of the fire-spotting parameter $\mu$ (left) and the diffusion coefficient $D$ (right) on $H_{\mathrm{ABL}}$.

drops back down to the ground, and the convective column is not high, and this affects both fire-spotting and turbulent transport of heat. The contour lines of the effective indicator function is plotted in Figure 4 . If the corresponding value of the effective indicator function reaches the threshold value $\phi_{\mathrm{e}}^{\text {th }}=0.5$, the grid point is marked as burned. Thus, these plots show the growth of the burning area after 37, 70 and 119 minutes after the ignition, respectively.

Case 2. Unstable atmosphere: $H_{\mathrm{ABL}}$ varies from $800 \mathrm{~m}$ to $2000 \mathrm{~m}$.

If an air parcel after displacement from the original height accelerates upward due to its buoyancy, such atmosphere is called unstable. Usually it is observed during the day and afternoon. The depth of the boundary layer varies due to season and clime classes. Usually the Köppen-Geiger climate classes are used to group the world-wide characteristics of the boundary layer [37. Thus, analysing seasonal mean diurnal cycle of ABL depth for climate class Dfb (Cold with warm summers and no dry season, during summer and winter) given in [37, one finds that the diurnal $H_{\mathrm{ABL}}$ varies between $1000 \mathrm{~m}$ and $1800 \mathrm{~m}$ during the summer, and between $600 \mathrm{~m}$ and $800 \mathrm{~m}$ during winter. For other climate classes, $H_{\mathrm{ABL}}$ may differ substantially, reaching up to $3000 \mathrm{~m}$. Fire front propagation for the unstable atmospheric conditions is shown in Figure 5

By comparing Figures 4 and 5 , we observe that the generation of secondary 
fires is more rapid in the unstable case, see both panels (b), such that the

burned area is larger and the merging of secondary fires is also more rapid, see both panels (c). Moreover, we highlight the remarkable ability of the model to generates not burned areas surrounded by the fire, see 5 . At the best knowledge of the authors, this is the first model generating these events.

The plots in Figure 6 show the lognormal distribution for selected values of the height of atmospheric boundary layer $H_{\mathrm{ABL}}$. According to the physical parametrisation proposed here, larger $H_{\mathrm{ABL}}$ corresponds to the higher probability of long jumps.

Figure 7 shows the total number of burned points simulated with several values of $H_{\mathrm{ABL}}$ at different times. After an initial period of quiescence with no visible contributions, the burned area rapidly increases as a power law. This power law seems to hold at stable atmospheric conditions, but the quiescence period ends first in unstable conditions such that the burned area during unstable conditions is always larger than during stable conditions.

In order to check if the rapid increasing of the burned area is mainly due to turbulence or fire-spotting we performed a series of simulations with fixed diffusion coefficient $D$, i.e., independent of the atmospheric conditions. The results for stable $\left(H_{\mathrm{ABL}}=100 \mathrm{~m}\right)$ and unstable $\left(H_{\mathrm{ABL}}=1000 \mathrm{~m}\right)$ atmospheric conditions are shown in Figures 8 and 9. All the parameters are set equal to the previous simulations, but with fixed $D=0.0387 \mathrm{~m}^{2} \mathrm{~s}^{-1}$. Note that this ${ }_{325}$ fixed value of $D$ is less than any other value followed by the dependency on the $H_{\mathrm{ABL}}$. Fire front propagates in the same way in both atmospheric conditions, displaying an equal number of secondary fires and equal burned area (see Figure 10. Thus, the atmospheric stability conditions affects the fire propagation mainly through the turbulent diffusion coefficient. Hence, comparing Figures 5 and 9 we observe that the number of independent fires is less during unstable conditions and by taking into account also Figures 7 and 10 we conclude that the atmospheric conditions affect the propagation of wildfires through the heat turbulent transfer. In particular, during unstable conditions we observe that turbulence pushes the front resulting into a faster propagation causing an in- 
that during unstable conditions the number of independent fires is less than during stable conditions in spite of the fact that the burned area is larger.

The viability of the proposed physical parametrisation is thus demonstrated through the numerical examples presented above. It is shown that the inclusion of the atmospheric stability conditions in terms of the ABL height may change the form and the speed of the fire front. However, the impact of atmospheric stability is significantly stronger for the main front behaviour by meaning of turbulent heat transfer than for the fire-spotting.

Let us now consider historical data on fires in Spain from 2012 to 2017 from The Earth Observing System Data and Information System (https:// earthdata.nasa.gov/earth-observation-data/near-real-time/firms/active-fire-data).

The database contains the information about the acquisition time but not about the atmospheric stability. In order to distinguish between stable and unstable conditions we consider these two extremely different cases [27]: diurnal ignitions during the summer months and nocturnal ignitions in winter months, of the historical data taken from two satellites with different resolution: MODIS C6 with resolution $1 \mathrm{~km}$ and VIIRS with resolution $375 \mathrm{~m}$ (see Table 2). More precise satellite VIIRS is capable to capture smaller wildfires reporting a higher number of ignitions, while MODIS C6 can identify only large enough wildfires.

Comparing the results in Table 2 one can find that the number of fires in unstable conditions captured by VIIRS satellite is almost 3 times larger than the observations by MODIS C6. In stable atmosphere the number of fires captured by VIIRS is usually more than 20 times larger. Taking into account the different resolution of the two satellites one can conclude that wildfires under unstable conditions are characterized by larger burning area, while under stable conditions the burning area is smaller with a significant increase in the number of wildfires.

As it is mentioned above, stable boundary layer is also known as nocturnal boundary layer [27, that allows to suppose that, in general, the stable atmo- 


\begin{tabular}{|c|rr|rr|}
\hline Year & \multicolumn{2}{|c|}{ MODIS C6 Data (1km) } & \multicolumn{2}{c|}{ VIIRS Data (375 m) } \\
& Stable & Unstable & Stable & Unstable \\
\hline 2012 & 138 & 1150 & 1170 & 3302 \\
2013 & 34 & 639 & 960 & 1580 \\
2014 & 16 & 361 & 803 & 1243 \\
2015 & 30 & 769 & 664 & 2167 \\
2016 & 38 & 672 & 1029 & 1895 \\
2017 & 64 & 671 & 1106 & 1866 \\
\hline
\end{tabular}

Table 2: Comparison of number of wildfires in stable that corresponds to winter night time, and unstable that corresponds to summer daylight time, atmosphere observed by satellites with different resolution. Historical data: Spain, 2012-2017 years.

layer is more unstable. Thus, the data collected from the satellites during the whole year is considered distinguishing the diurnal and nocturnal fires (see Table 3).

Taking into account that only large enough burning areas may be captured by the MODIS C6 satellite, while VIIRS is able to identify smaller ignitions, data from Table 2 can be interpreted as follows. During the day wind is more erratic and strong that cause more unpredictable behaviour of the wildfire. During the night the main front may propagate slower but many new ignitions may occur.

Distribution of day/night fires in 2016 and 2017 captured by satellites depending on date is presented in Figures 11 and 12. Most part of fires accounts for summer time that is caused by the high temperatures and the dry and hot air. However, huge number of night fires was registered in October, 2017, mainly in Galicia and Asturias. As it is known from the official statistical data (http://www.mapama.gob.es), more than $50 \%$ of new ignitions in Spain are deliberate. We expect that the most of them occur during the night, that also explains the data presented in Table 3 and blue peaks of the graph in Figures 11 and 12

During the day the number of ignitions is smaller but the burning area 


\begin{tabular}{|c|cc|cc|}
\hline \multirow{2}{*}{ Year } & \multicolumn{2}{|c|}{ MODIS C6 Data $(1 \mathrm{~km})$} & \multicolumn{2}{c|}{ VIIRS Data $(375 \mathrm{~m})$} \\
& Day & Night & Day & Night \\
\hline 2012 & $4240(68.5 \%)$ & $1946(31.5 \%)$ & $8430(39.4 \%)$ & $12945(60.6 \%)$ \\
2013 & $2631(76.2 \%)$ & $821(23.8 \%)$ & $6261(45.3 \%)$ & $7575(54.7 \%)$ \\
2014 & $2181(83.8 \%)$ & $422(16.2 \%)$ & $5400(42.8 \%)$ & $7228(57.2 \%)$ \\
2015 & $2317(73.0 \%)$ & $857(27.0 \%)$ & $5547(41.8 \%)$ & $7723(58.2 \%)$ \\
2016 & $2328(72.5 \%)$ & $884(27.5 \%)$ & $7906(42.9 \%)$ & $5942(57.1 \%)$ \\
2017 & $3578(69.0 \%)$ & $1606(31.0 \%)$ & $8288(42.0 \%)$ & $11470(58.0 \%)$ \\
\hline
\end{tabular}

Table 3: Number and percentage of day and night fires in Spain. Historical data 2012-2017 years.

is larger, since the percentage of the daytime fires obtained by MODIS C6 is higher than the percentage of night-time fires and vice-a-versa for the VIIRS data. Thus, these data can be interpret similarly to the results obtained from the simulations, during the daytime the higher ABL layer causes an higher convection cell, that apart from the larger travel distance of the firebrand, increases also the turbulent transport. This results into a faster fire front propagation and more rapid merging of the spotting fires. Consequently, it leads to the higher burning area and a smaller number of independent secondary fires during the daytime. As it is shown above, such scenario can be modelled by the proposed model varying the $H s u b A B L$, that allows for adjusting the proposed model to different climatic classes.

\section{Conclusions}

Fire-spotting is a important factor for risk increase in wildfires. Our study reveals that atmospheric stability conditions affect the propagation of the fire front, but in spite of the fact that they have a direct effect on the maximum landing distance of firebrands, their effects on the turbulent heat transport governs the evolution of the wildfire. In particular, unstable conditions (in general occurring during the daytime) boost the number of fire-spotting generated fires 
at small elapsed times as well as the strength of turbulence leading to rapid merging and the formation of unburned islands surrounded by the fire. At large times, during unstable conditions the burned area is larger in spite of a lower number of independent secondary ignitions with respect to the cases during stable conditions (in general occurring during the night-time). This behaviour results mainly due to the effect of the depth of the ABL on turbulent heat diffusion. In fact, a stronger turbulence pushes the fire front generating a rapid propagation causing also a more rapid merging of independent fires. The analysis of data from MODIS C6 and VIIRS satellites shows that this qualitative behaviour is observed from the real data.

Actually, atmospheric stability is not the only factor that affects wildfires. Many of other natural factors including surround vegetation, topography, etc., and social factors, such fire prevention strategies and land use, can increase the risk of damages by wildfires on the ecosystems and for human lives and properties. The proposed parametrisation allows for taking into account also an other important natural factors as the flame geometry. Flame geometry includes environmental and fuel conditions, including fuel density, ambient temperature, fire intensity and gravity. These factors will be implemented and their role on fire-spotting generated fires investigated in the second part of the present study.

\section{Appendix: Approximated lofting height for firebrands}

The motion if firebrands lofted by a smoke column is here assumed to be similar to the motion if inertial particles into a fluid flow.

Let $v, u$ and $u^{*}$ be the velocity of the inertial particle, the fluid velocity and the fluid velocity along the inertial particle trajectory. Moreover, let $\tau_{p}$ and $\tau_{L}$ be the inertial particle and the fluid-Lagrangian timescale, respectively, if it holds

$$
\tau_{\mathrm{p}} \ll \tau_{\mathrm{L}}
$$

by using Lagrangian stochastic models, it has been shown against data that for 
practical purposes the following approximation works [38, 39]

$$
v \simeq u^{*}-\tau_{\mathrm{p}} g,
$$

where $g$ is the gravitational acceleration.

Let us consider now the fluid velocity field $u$ as the smoke velocity field. We can compute the maximum height as follows:

$$
H=\int_{0}^{T} v d t \simeq \int_{0}^{T} u^{*} d t-\tau_{\mathrm{p}} g \int_{0}^{T} d t \simeq H^{*}-\tau_{\mathrm{p}} g T,
$$

Let $V_{S}$ be the Stokes terminal velocity, an estimation of $T$ is

$$
T=\frac{H}{V_{S}} .
$$

The correlation time of the smoke (fluid) velocity field $u$ is longer than the correlation time of the velocity field $u^{*}$, let us say $\tau_{\mathrm{L}}=\vartheta \tau^{*}>\tau^{*}$ 38. Let $H_{\text {smoke }}$ be the maximum height reached by a smoke particle, then

$$
H_{\text {smoke }}=\vartheta H^{*}>H^{*} .
$$

Plugging (31) and (32) into (30) we have

$$
H=\frac{V_{S}}{V_{S}+\tau_{p} g} \frac{H_{\text {smoke }}}{\vartheta} .
$$

Finally, remembering that

$$
V_{S}=\frac{2}{9} \frac{\rho_{\mathrm{f}}-\rho}{\mu_{\mathrm{visc}}} g r^{2}, \quad \tau_{\mathrm{p}}=\frac{1}{18} \frac{\rho_{\mathrm{f}}}{\mu_{\mathrm{visc}}} r^{2},
$$

where $\rho_{\mathrm{f}}$ and $\rho$ are the firebrand and the smoke density, $\mu_{\mathrm{visc}}$ is the dynamic viscosity and $r$ is the firebrand radius, adopting the approximated value $\vartheta=$ 2 , i.e., the decorrelation of the smoke velocity field along the inertial particle trajectory is two times faster than along its own smoke passive trajectory, and that $\rho_{\mathrm{f}} \gg \rho$, the maximum lofting height for firebrands emerges to be

$$
H \simeq 0.4 H_{\text {smoke }}
$$




\section{Nomenclature}

Roman symbols 


\begin{tabular}{|c|c|}
\hline$C_{\mathrm{d}}$ & Drag coefficient, $C_{\mathrm{d}}=0.45$ \\
\hline$D$ & Diffusion coefficient, $\mathrm{m}^{2} \mathrm{~s}^{-1}$ \\
\hline$d$ & Unit depth of the combustion zone, $d=1 \mathrm{~m}$ \\
\hline$G(\mathbf{x}-\overline{\mathbf{x}} ; t)$ & Isotropic bi-variate Gaussian function \\
\hline$g$ & Gravitational acceleration, $g=9.81 \mathrm{~ms}^{-2}$ \\
\hline$F r$ & Froude number \\
\hline$f(\mathbf{x} ; t \mid \overline{\mathbf{x}})$ & $\begin{array}{l}\text { Probability density function of the displacement of the active flame holders } \\
\text { around the average position } \overline{\mathbf{x}}\end{array}$ \\
\hline$H$ & Maximum loftable height of a firebrand (m) \\
\hline$H_{\mathrm{ABL}}$ & Height of the atmospheric boundary layer, $H_{\mathrm{ABL}}=100 \ldots 2000 \mathrm{~m}$ \\
\hline$H_{\mathrm{c}}$ & Height of convection cell $(\mathrm{m})$ \\
\hline$H_{\text {smoke }}$ & Maximum height reached by a smoke particle (m) \\
\hline$I_{\mathrm{f}}$ & Fireline intensity, $I_{\mathrm{f}}=2 \cdot 10^{4} \mathrm{Wm}^{-1}$ \\
\hline$\ell$ & Firebrand landing distance (m) \\
\hline$\ell_{0}$ & Minimum travel distance $(\mathrm{m})$ \\
\hline$\ell_{\max }$ & Maximum travel distance $(\mathrm{m})$ \\
\hline$N_{0}^{2}$ & Brunt-Väisälä frequency at the current height, $N_{0}^{2}=2.5 \cdot 10^{-4} \mathrm{~s}^{-2}$ \\
\hline$N_{\mathrm{FT}}^{2}$ & Brunt-Väisälä frequency in the free troposphere, $N_{\mathrm{FT}}^{2}=2.789 \cdot 10^{-4} \mathrm{~s}^{-2}$ \\
\hline $\mathbf{n}$ & Normal to the fire front \\
\hline$P_{f 0}$ & Ratio of reference fire power, $P_{f 0}=1 \mathrm{MWm}^{-2}$ \\
\hline$q(l)$ & Firebrand distribution \\
\hline$r$ & Firebrand radius $(\mathrm{m})$ \\
\hline$R a$ & Rayleigh number \\
\hline$T$ & Lofting time $(s)$ \\
\hline$t$ & Time $(s)$ \\
\hline$U$ & Mean wind velocity $U=4.47 \mathrm{~ms}^{-1}$ \\
\hline$u$ & Fluid velocity $\left(\mathrm{ms}^{-1}\right)$ \\
\hline$u^{*}$ & Fluid velocity along the inertial particle trajectory $\left(\mathrm{ms}^{-1}\right)$ \\
\hline$V_{\mathrm{ROS}}(\mathbf{x}, t)$ & Rate of spread $\left(\mathrm{ms}^{-1}\right)$ \\
\hline$V_{\mathrm{S}}$ & Stokes terminal velocity $\left(\mathrm{ms}^{-1}\right)$ \\
\hline$v$ & Velocity of the inertial particle $\left(\mathrm{ms}^{-1}\right)$ \\
\hline $\mathrm{x}$ & Spatial coordinates \\
\hline$z_{\mathrm{p}}$ & $p$-th percentile that can be estimated from the $z$-tables, $p=67, z_{\mathrm{p}}=0.45$ \\
\hline
\end{tabular}


$z_{\mathrm{p}}^{0} \quad$ percentile that corresponds to the minimum travel distance of the firebrand

\section{Greek symbols}

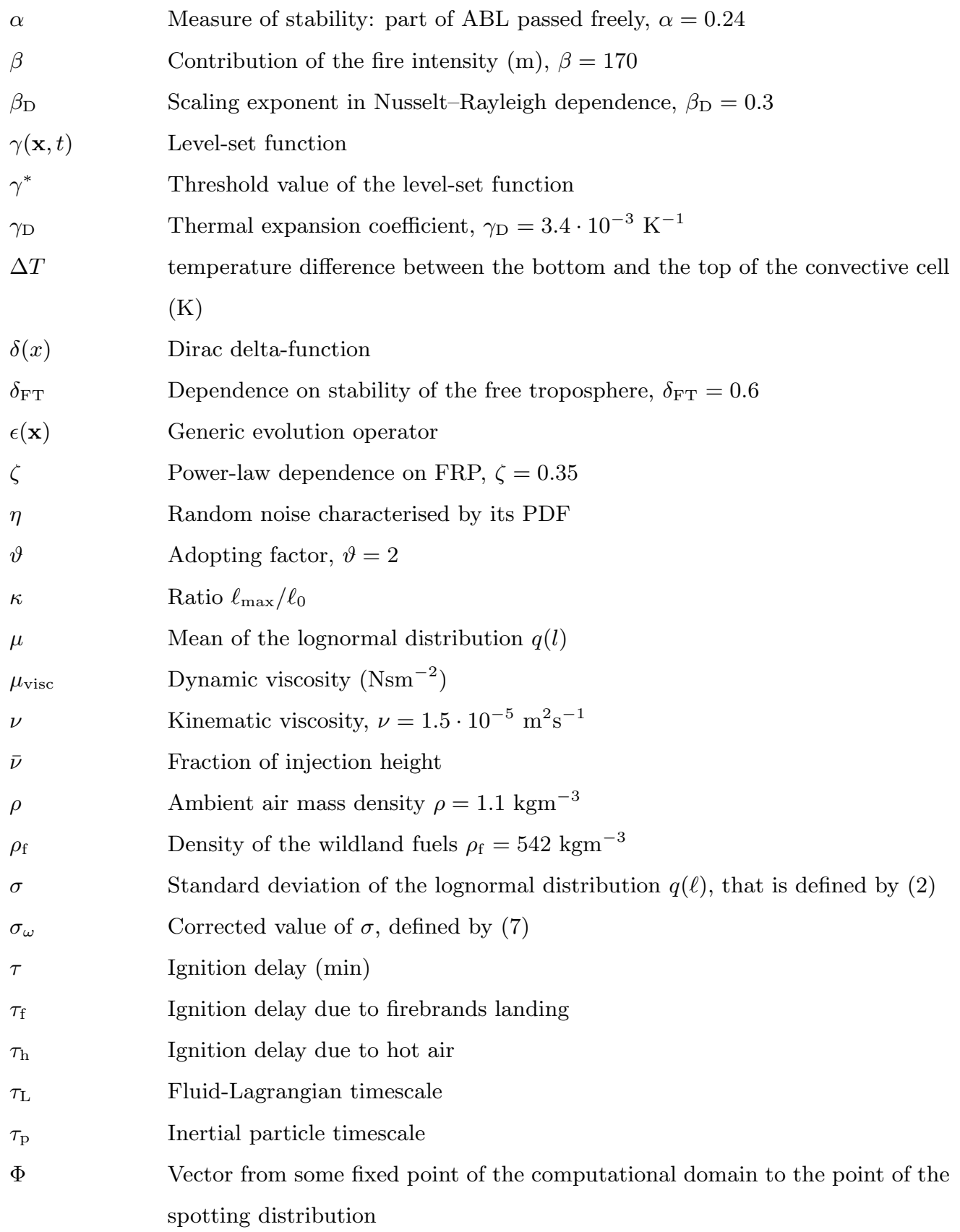




$\begin{array}{ll}\phi(\mathbf{x}, t) & \text { Indicator function, } \phi \in\{0,1\} \\ \phi_{\mathrm{e}}(\mathbf{x}, t) & \text { Effective indicator function, } \phi_{\mathrm{e}} \in[0,1] \\ \phi_{\mathrm{e}}^{t h} & \text { Threshold value of the effective indicator function } \\ \chi & \text { Thermal diffusivity of the ambient air, } \chi=2 \cdot 10^{-6} \mathrm{~m}^{2} \mathrm{~s}^{-1} \\ \psi(\mathbf{x}, t) & \text { Amount of heat accumulated in time } t \\ \Omega(t) & \text { Burnt area } \\ \omega & \text { Angle between the mean wind and vector to the point, where the lognormal } \\ & \text { distribution is calculated } \\ \omega_{0} & \text { Critical value of } \omega\end{array}$

\section{Code availability}

The code LSFire+ is developed in C and Fortran where the model RandomFront 2.3 acts as a post-processing routine at each time step in a LSM code for the front propagation implemented by the means of LSMLIB [40] and the ROS is computed by using the library FireLib [41]. The numerical library LSMLIB is written in Fortran2008/OpenMP and propagates the fire-line through standard algorithms for the LSM, including also Fast Marching Method algorithms. Implementation of RandomFront 2.3 in LSFire+ is freely available at the official git repository of BCAM, Bilbao, https://gitlab.bcamath.org/atrucchia/ randomfront-wrfsfire-lsfire.

440 Simulations with LSFire+ are perfomed over the cluster HYPATIA of BCAM, Bilbao, using OpenMP shared memory parallelism, running over 24 cores inside of a Intel(R) Xeon(R) CPU E5-2680 v3 2.50GHz node with 128GB RAM. The computational time for each simulation, that spanned 140 minutes of physical time, was about 45 minutes.

The $80 \%$ of the computational cost is due to the post-processing routine RandomFront 2.3. This computational time can be reduced in the future through a further code optimisation. 


\section{Acknowledgements}

This research is supported by the Basque Government through the BERC

450

and Competitiveness MINECO through BCAM Severo Ochoa excellence accreditations SEV-2013-0323 and SEV-2017-0718 and project MTM2016-76016R "MIP", and by the PhD grant "La Caixa 2014".

\section{References}

[3] A. L. Sullivan, Inside the inferno: Fundamental processes of wildland fire behaviour. Part 1: Combustion chemistry and heat release, Curr. Forestry Rep. 3 (2017) 132-149.

[4] A. L. Sullivan, Inside the inferno: Fundamental processes of wildland fire 470 behaviour. Part 2: Heat transfer and interaction, Curr. Forestry Rep. 3 (2017) 150-171.

[5] A. C. Fernandez-Pello, Wildland fire spot ignition by sparks and firebrands, Fire Saf. J. 91 (2017) 2-10. 
[6] G. Pagnini, A. Mentrelli, Modelling wildland fire propagation by tracking random fronts, Nat. Hazards Earth Syst. Sci. 14 (8) (2014) 2249-2263.

[7] I. Kaur, A. Mentrelli, F. Bosseur, J.-B. Filippi, G. Pagnini, Turbulence and fire-spotting effects into wild-land fire simulators, Commun. Nonlinear Sci. Numer. Simul. 39 (2016) 300-320.

[8] J. A. Sethian, P. Smereka, Level set methods for fluid interfaces, Annu. Rev. Fluid Mech. 35 (2003) 341-372.

[9] V. Mallet, D. Keyes, F. Fendell, Modeling wildland fire propagation with level set methods, Comput. Math. Appl. 57 (7) (2009) 1089-1101.

[10] J. Mandel, J. D. Beezley, A. K. Kochanski, Coupled atmosphere-wildland fire modeling with WRF 3.3 and SFIRE 2011, Geosci. Model. Dev. 4 (2011) 591-610.

[11] C. Tarifa, P. del Notario, F. Moreno, On flight paths and lifetimes of burning particles of wood, in: Tenth Symposium on Combustion, 1721 August 1964, Cambridge, UK, The Combustion Institute: Pittsburgh, PA, 1965, pp. 1021-1037.

[12] F. A. Albini, Spot fire distance from burning trees: a predictive model, Technical Report INT-56, U.S. Department of Agriculture Forest Service Intermountain Forest and Range Experiment Station (1979).

[13] F. A. Albini, Potential spotting distance from wind-driven surface fires, Research Paper INT-309, U.S. Department of Agriculture Forest Service Intermountain Forest and Range Experiment Station (1983).

[14] M. Finney, Farsite: fire area simulator - model development and evaluation, Research Paper RMRS-RP-4, USDA Forest Service, Rocky Mountain Research Station, Ogden, Utah (1998).

[15] C. Tymstra, R. Bryce, B. Wotton, S. Taylor, O. Armitage, evelopment and structure of Prometheus: the Canadian wild land fire growth simulation 
model, Information Report NOR-X-417, Canadian Forest Service, Northern Forestry Centre (2010).

[16] H. H. Wang, Analysis on downwind distribution of firebrands sourced from a wildland fire, Fire Technol. 47 (2011) 321-340.

[17] N. Sardoy, J. L. Consalvi, B. Porterie, A. C. Fernandez-Pello, Modeling transport and combustion of firebrands from burning trees, Combust. Flame 150 (2007) 151-169.

[18] N. Sardoy, J. Consalvi, A. Kaiss, A. Fernandez-Pello, B. Porterie, Numerical study of ground-level distribution of firebrands generated by line fires, Combust. Flame 154 (3) (2008) 478-488.

[19] A. Tohidi, N. Kaye, W. Bridges, Statistical description of firebrand size and shape distribution from coniferous trees for use in Monte Carlo simulations of firebrand flight distance, Fire Saf. J. 77 (2015) 21-35.

[20] A. Tohidi, N. B. Kaye, Stochastic modeling of firebrand shower scenarios, Fire Saf. J. 91 (2017) 91-102.

[21] J. Martin, T. Hillen, The spotting distribution of wildfires, Applied Sciences $6(177)$.

[22] I. Kaur, G. Pagnini, Fire-spotting modelling and parametrisation for wildland fires, in: S. Sauvage, J. M. Sánchez-Pérez, A. E. Rizzoli (Eds.), Proceedings of the 8th International Congress on Environmental Modelling and Software (iEMSs2016); Toulouse, France, 10-14 July (2016), 2016, pp. 384-391, ISBN: 978-88-9035-745-9.

[23] M. Sofiev, T. Ermakova, R. Vankevich, Evaluation of the smoke-injection height from wild-land fires using remote-sensing data, Atmospheric Chem. Phys. 12 (4) (2012) 1995-2006.

[24] M. E. Alexander, Calculating and interpreting forest fire intensities, Can. J. Bot. 60 (1982) 349-357. 
[25] K. Himoto, T. Tanaka, Transport of disk-shaped firebrands in a turbulent boundary layer, The Eighth International Symposium on Fire Safety Science, 1823 September 2005, Beijing, China, edited by D. Gottuk and B. Lattimer (2005) 433-444.

[26] H.-H. Wang, Analysis on downwind distribution of firebrands sourced from a wildland fire, Fire Technol. 47 (2) (2011) 321-340.

[27] R. B. Stull, An Introduction to Boundary Layer Meteorology, Kluwer Academic Publishers, 1988.

[28] J. L. Dupuy, J. Marechal, D. Portier, D. Morvan, Fires from a cylindrical forest fuel burner: combustion dynamics and flame properties, Combust. Flame 135 (2003) 2029-2036.

[29] K. Mphale, M. Heron, Microwave measurement of electron density and collision frequency of a pine fire, J. Phys. D: Appl. Phys. 40 (2007) 28182825 .

[30] J. J. Niemela, L. Skrbek, K. R. Sreenivasan, R. J. Donnelly, Turbulent convection at very high Rayleigh numbers, Nature 404 (2000) 837-840.

[31] J. J. Niemela, K. R. Sreenivasan, Turbulent convection at high Rayleigh numbers and aspect ratio 4, J. Fluid Mech. 557 (2006) 411-422.

[32] X.-Z. Wu, A. Libchaber, Scaling relations in thermal turbulence: The aspect-ratio dependence, Phys. Rev. A 45 (1992) 842-845.

[33] R. G. Rehm, R. J. McDermott, Fire-front propagation using the level set method, Tech.note 1611, Natl. Inst. Stand. Technol. (2009).

[34] G. Pagnini, L. Massidda, The randomized level-set method to model turbulence effects in wildland fire propagation, in: D. Spano, V. Bacciu, M. Salis, C. Sirca (Eds.), Modelling Fire Behaviour and Risk. Proceedings of the International Conference on Fire Behaviour and Risk. ICFBR 2011, Alghero, Italy, October 4-6 2011, 2012, pp. 126-131, ISBN 978-88-904409-7-7. 
[35] G. Pagnini, L. Massidda, Modelling turbulence effects in wildland fire propagation by the randomized level-set method, Tech. Rep. 2012/PM12a, CRS4, Pula (CA), Sardinia, Italy, revised version: August 2014, available at: http://publications.crs4.it/pubdocs/2012/PM12a/pagnini_massiddalevelset.pdf and arXiv:1408.6129 (July 2012).

[36] A. N. Shiryaev, Probability-1, 3rd Edition, Graduate Texts in Mathematics, Springer, 2016.

[37] E. L. McGrath-Spangler, A. Molod, Comparison of geos-5 agcm planetary boundary layer depths computed with various definitions, Atmospheric Chemistry and Physics 14 (13) (2014) 6717-6727.

[38] J. D. Wilson, Trajectory models for heavy particles in atmospheric turbulence: Comparison with observations, J. Appl. Meteor. 39 (2000) 18941912 .

[39] T. Bouvet, J. D. Wilson, A. Tuzet, Observations and modeling of heavy particle deposition in a windbreak flow, J. Appl. Meteorol. Climatol. 45 (2006) 1332-1349.

[40] K. T. Chu, M. Prodanović, Level set method library (LSMLIB)http://ktchu. serendipityresearch.org/software/lsmlib/.

[41] C. D. Bevins, Firelib: User manual and technical reference, Tech. rep., US Forest Service, Missoula Fire Sciences Laboratory, Fire Behavior Research Work Unit Systems for Environmental Management (1996).

URL https://www.frames.gov/catalog/935 


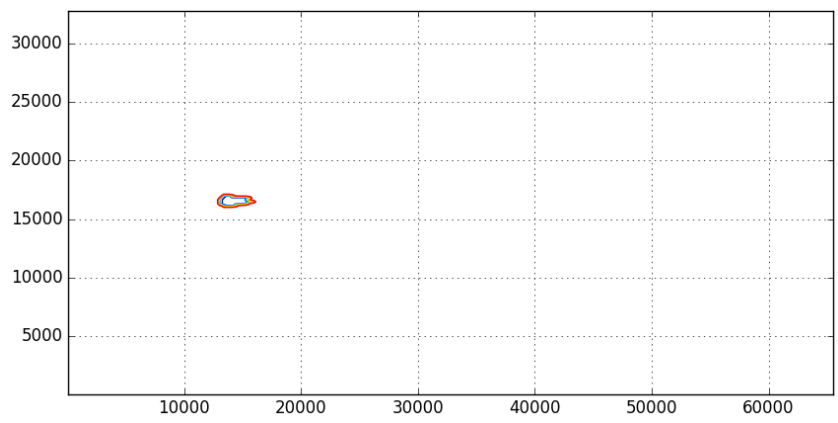

(a) $t=37 \mathrm{~min}$.

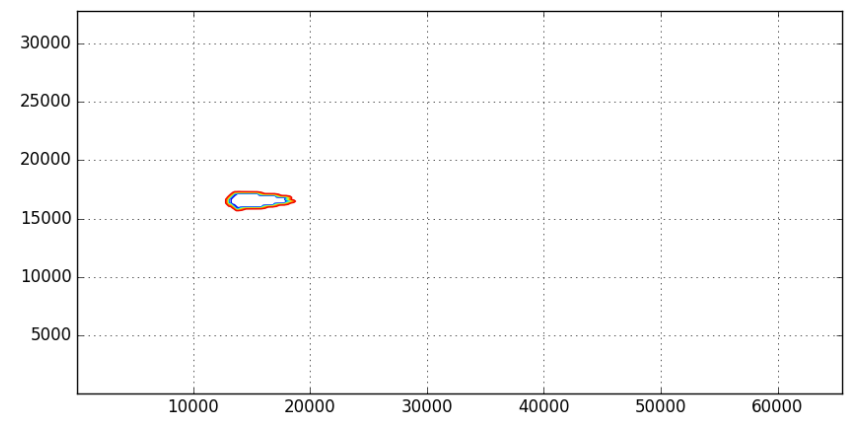

(b) $t=70 \mathrm{~min}$.

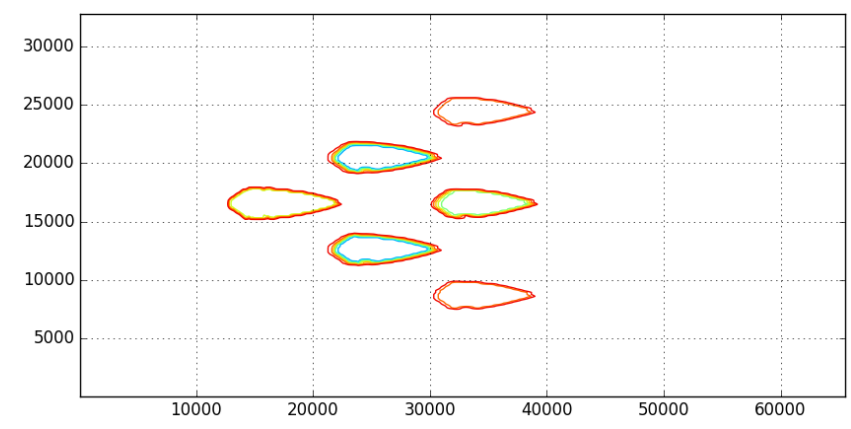

(c) $t=119 \mathrm{~min}$.

Figure 4: Fire front propagation during stable atmospheric boundary conditions: $H_{\mathrm{ABL}}=100$ $\mathrm{m}, \mu=4.0325 \mathrm{~m}, D=0.0533 \mathrm{~m}^{2} \mathrm{~s}^{-1}$. 


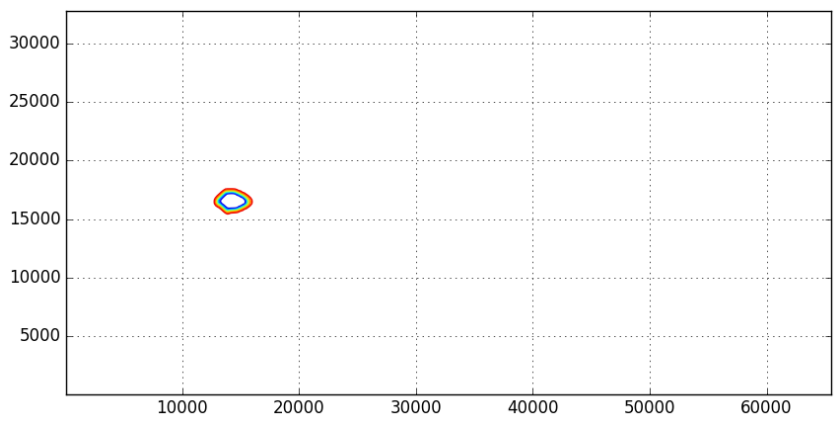

(a) $t=37 \mathrm{~min}$.

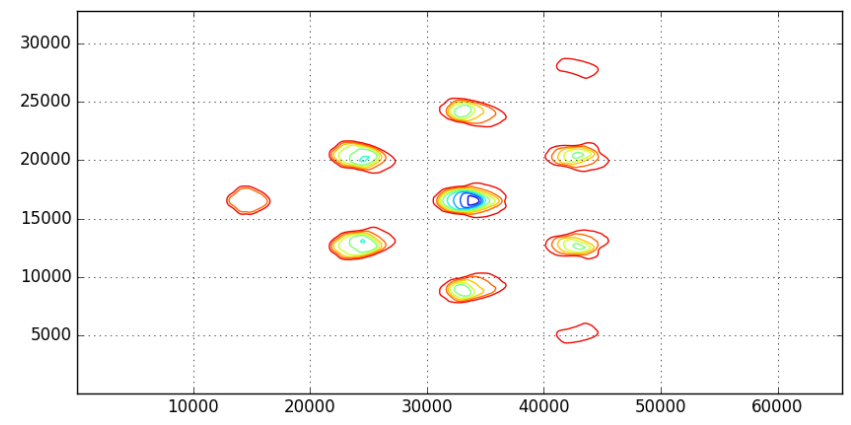

(b) $t=53 \mathrm{~min}$.

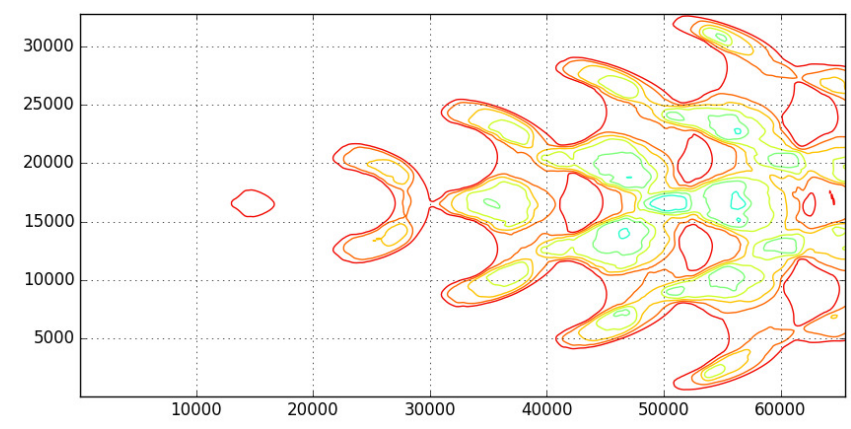

(c) $t=70 \mathrm{~min}$.

Figure 5: Fire front propagation during unstable atmospheric boundary conditions: $H_{\mathrm{ABL}}=$ $1000 \mathrm{~m}, \mu=7.9411 \mathrm{~m}, D=0.4238 \mathrm{~m}^{2} \mathrm{~s}^{-1}$. 


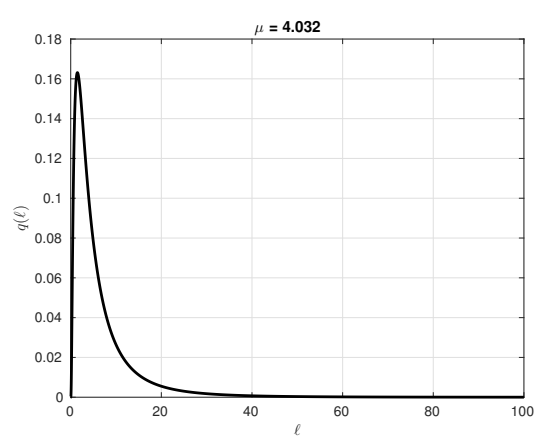

(a) $H_{\mathrm{ABL}}=100 \mathrm{~m}$.

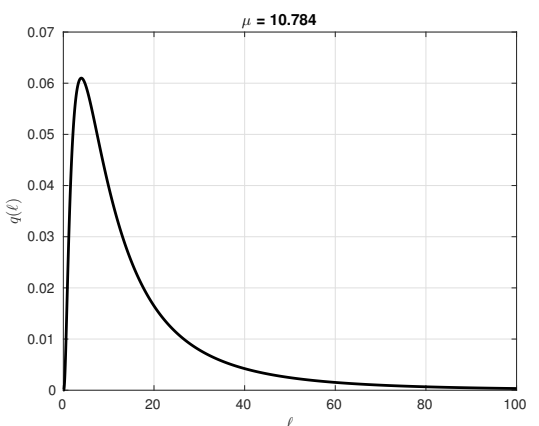

(b) $H_{\mathrm{ABL}}=1000 \mathrm{~m}$.

Figure 6: PDFs of the lognormal distribution for various values of $H_{\mathrm{ABL}}$, that corresponds to the stable and unstable atmospheric conditions.

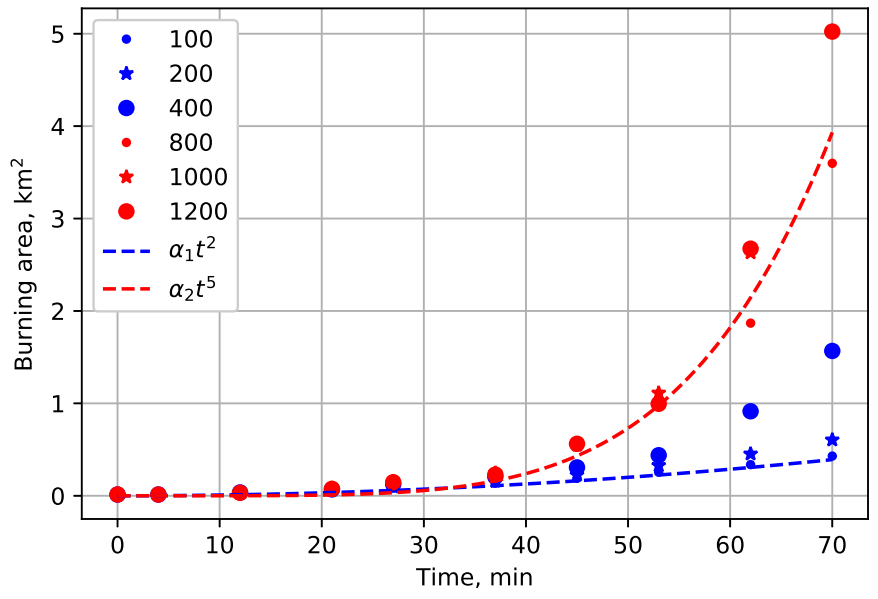

Figure 7: A comparison of the burned area in different time moments in stable (blue) and unstable (red) atmospheric boundary conditions. Simulations are provided for various values of $H_{\mathrm{ABL}}$ from $100 \mathrm{~m}$ to $1200 \mathrm{~m}$. 


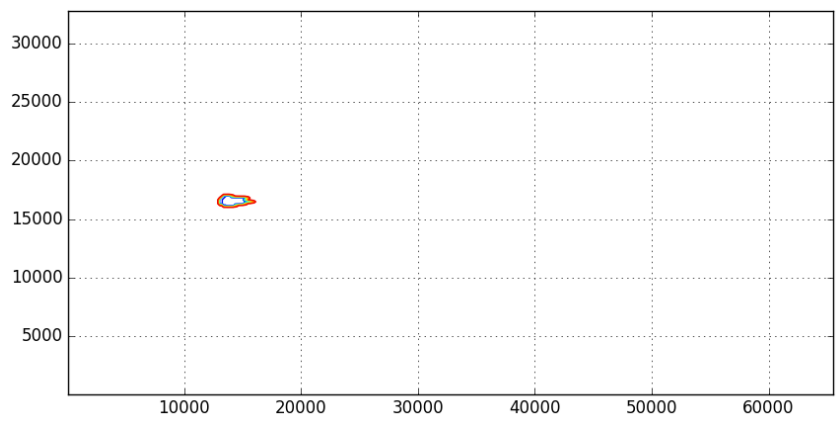

(a) $t=37 \mathrm{~min}$.

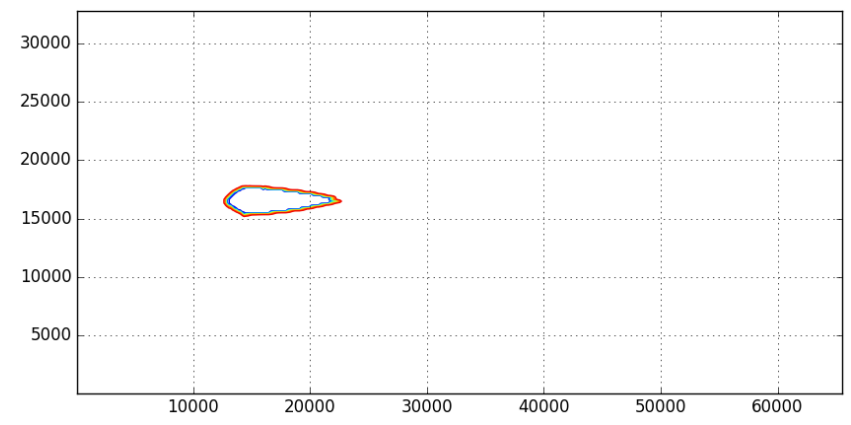

(b) $t=119 \mathrm{~min}$.

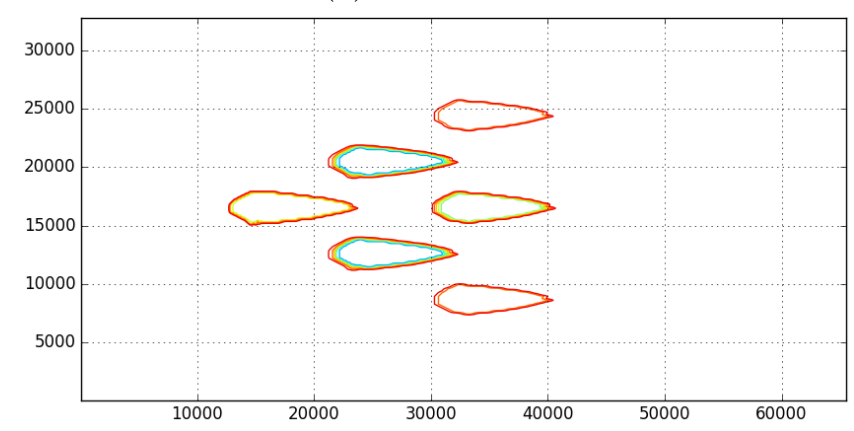

(c) $t=135 \mathrm{~min}$.

Figure 8: Fire front propagation during the night (Stable atmospheric boundary conditions: $\left.H_{\mathrm{ABL}}=100 \mathrm{~m}, \mu=4.0325 \mathrm{~m}, D=0.0387 \mathrm{~m}^{2} \mathrm{~s}^{-1}\right)$. 


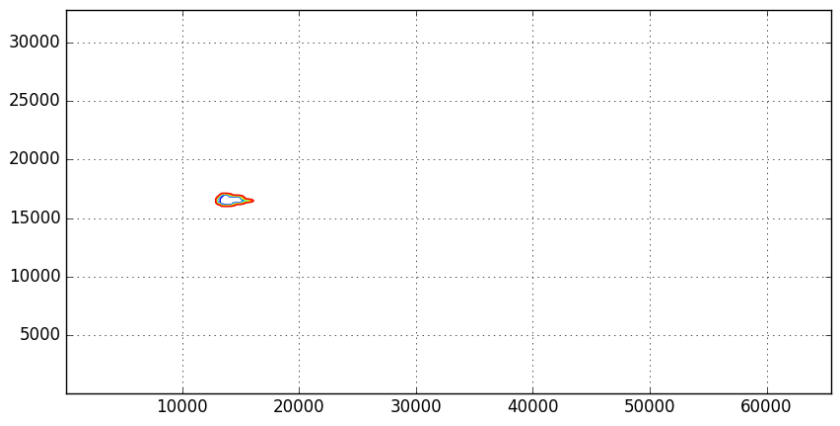

(a) $t=37 \mathrm{~min}$.

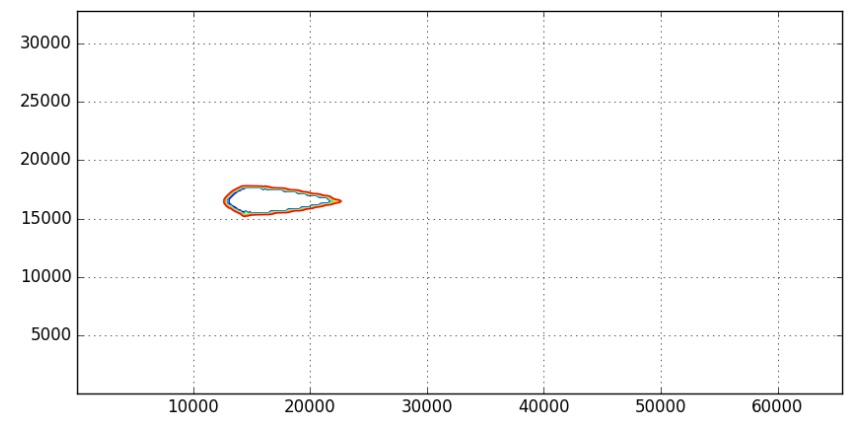

(b) $t=119 \mathrm{~min}$.

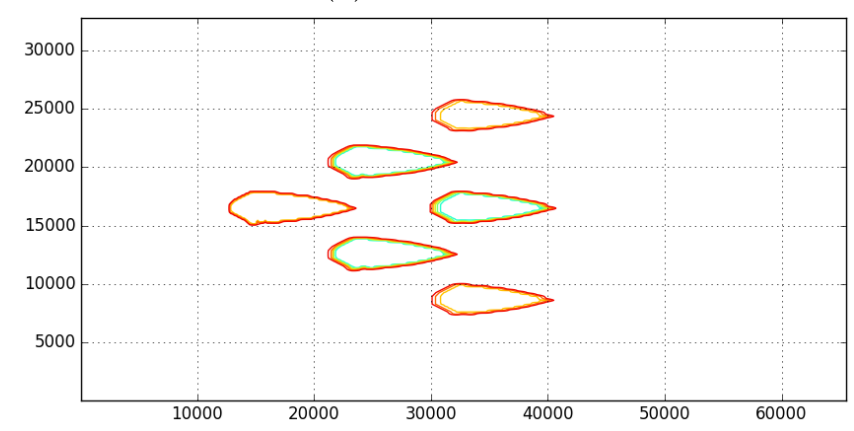

(c) $t=135 \mathrm{~min}$.

Figure 9: Fire front propagation during the daylight (Unstable atmospheric boundary conditions: $\left.H_{\mathrm{ABL}}=1000 \mathrm{~m}, \mu=7.2304 \mathrm{~m}, D=0.0387 \mathrm{~m}^{2} \mathrm{~s}^{-1}\right)$. 


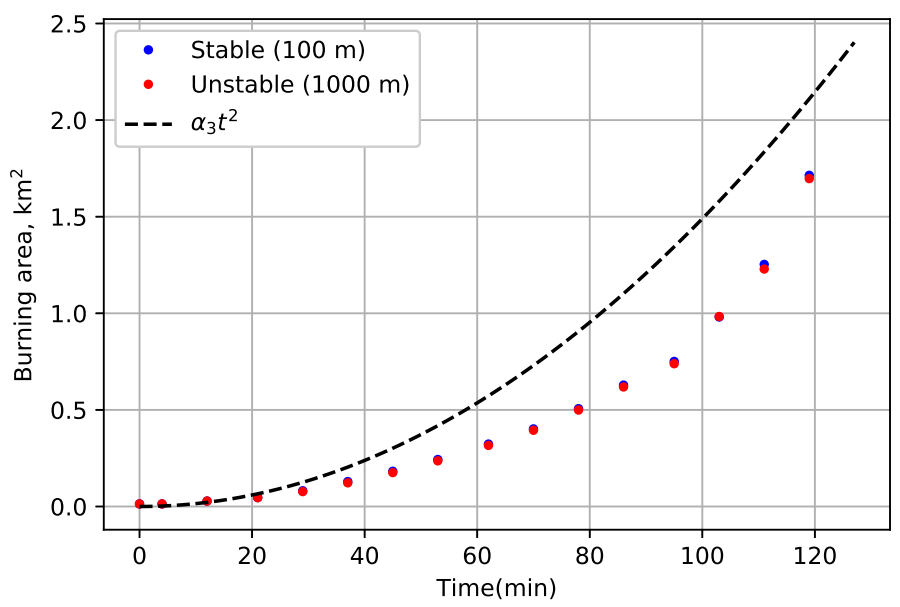

Figure 10: A comparison of the total burning area in time in stable (blue) and unstable (red) atmospheric boundary conditions in simulations with fixed diffusion coefficient $D=0.0387$ $\mathrm{m}^{2} \mathrm{~s}^{-1}$. 


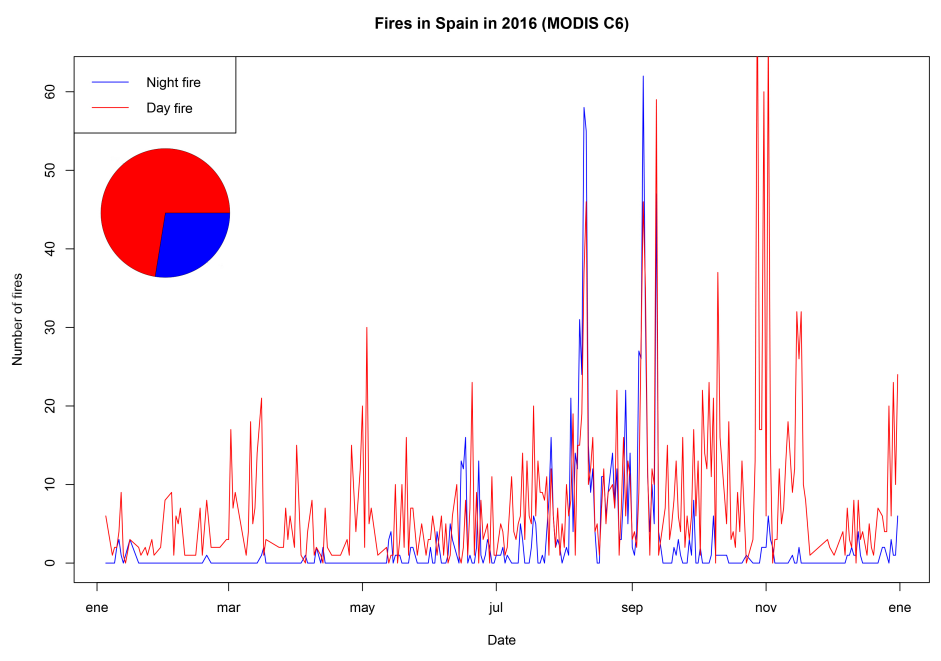

(a) MODIS C6

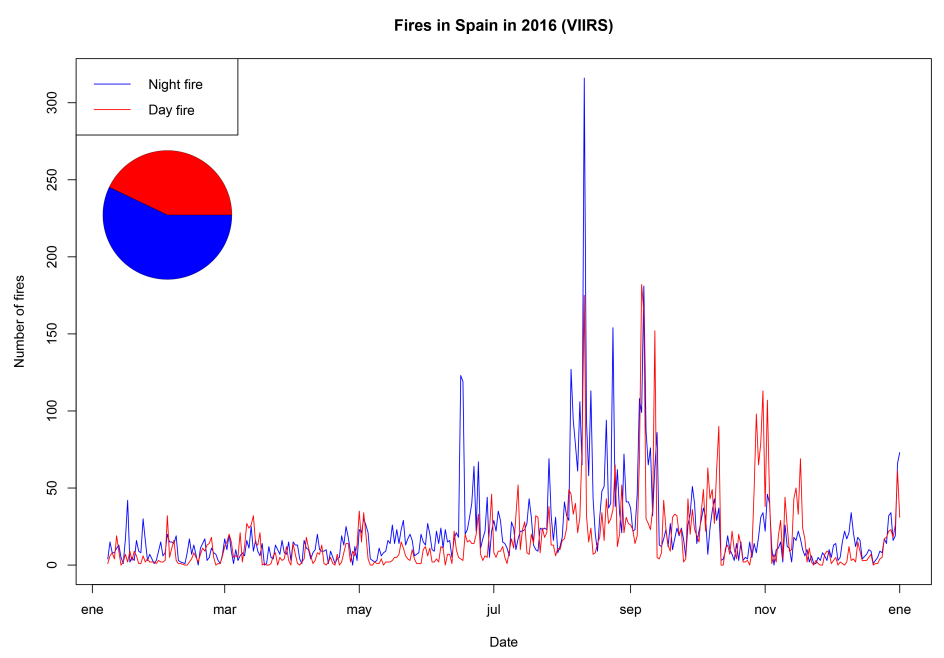

(b) VIIRS

Figure 11: Number of day and night fires during 2016 in Spain. 


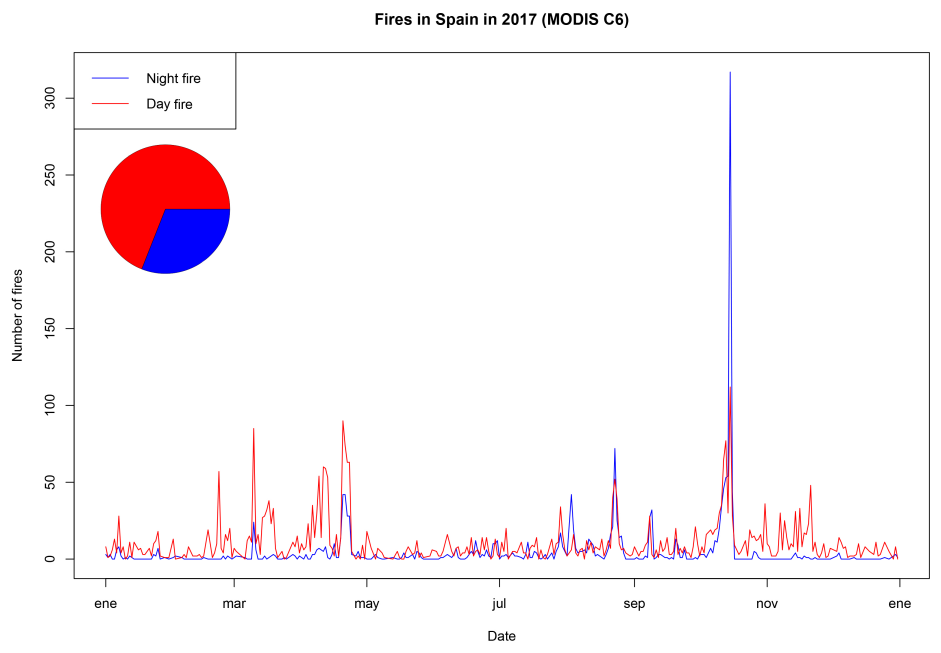

(a) MODIS C6

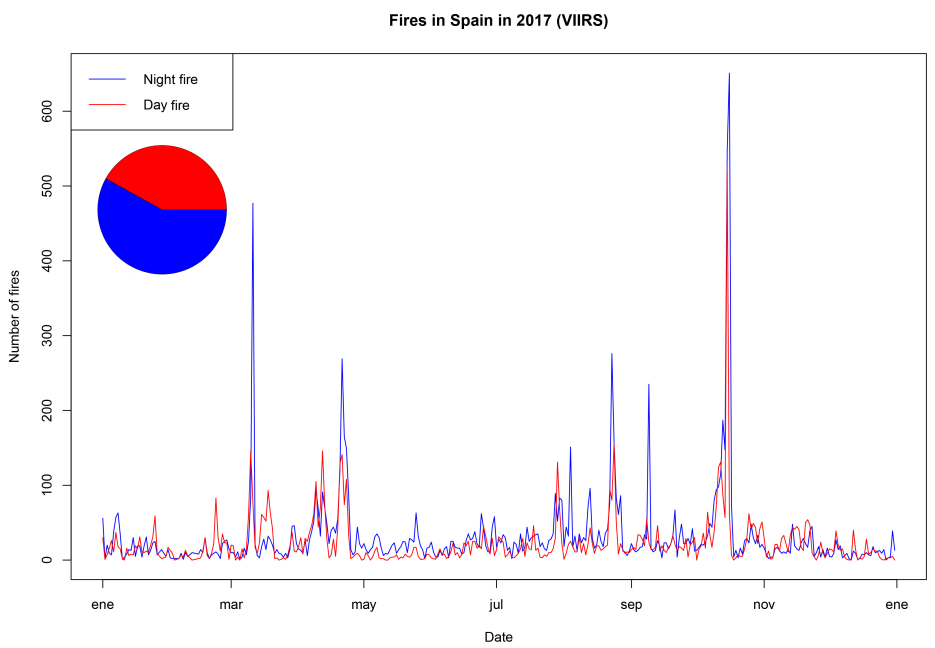

(b) VIIRS

Figure 12: Number of day and night fires during 2017 in Spain. 